\title{
Revealing the behavior of soliton buildup in a mode-locked laser
}

\author{
Xueming Liu ${ }^{\mathrm{a}, \mathrm{b}, \mathrm{c}, \star}$ and Yudong Cui ${ }^{\mathrm{a}}$ \\ aZhejiang University, College of Optical Science and Engineering, State Key Laboratory of Modern Optical Instrumentation, Hangzhou, China \\ ${ }^{b}$ Nanjing University of Aeronautics and Astronautics, Institute for Advanced Interdisciplinary Research, Nanjing, China \\ 'Hunan University of Science and Technology, School of Physics and Electronic Science, Xiangtan, China
}

\begin{abstract}
Real-time spectroscopy based on an emerging time-stretch technique can map the spectral information of optical waves into the time domain, opening several fascinating explorations of nonlinear dynamics in mode-locked lasers. However, the self-starting process of mode-locked lasers is quite sensitive to environmental perturbation, which causes the transient behaviors of lasers to deviate from the true buildup process of solitons. We optimize the laser system to improve its stability, which suppresses the $Q$-switched lasing induced by environmental perturbation. We, therefore, demonstrate the first observation of the entire buildup process of solitons in a mode-locked laser, revealing two possible pathways to generate the temporal solitons. One pathway includes the dynamics of raised relaxation oscillation, quasimode-locking stage, spectral beating behavior, and finally the stable single-soliton mode-locking. The other pathway contains, however, an extra transient bound-state stage before the final single-pulse modelocking operation. Moreover, we propose a theoretical model to predict the buildup time of solitons, which agrees well with the experimental results. Our findings can bring real-time insights into ultrafast fiber laser design and optimization, as well as promote the application of fiber laser.
\end{abstract}

Keywords: fiber laser; mode-locking; self-starting process; relaxation oscillation; real-time spectroscopy.

Received Oct. 22, 2018; accepted for publication Dec. 25, 2018; published online Jan. 28, 2019.

(C) The Authors. Published by SPIE and CLP under a Creative Commons Attribution 4.0 Unported License. Distribution or reproduction of this work in whole or in part requires full attribution of the original publication, including its DOI.

[DOI: 10.1117/1.AP.1.1.016003]

\section{Introduction}

Transient phenomena and dynamics are important characteristics of numerous nonlinear systems. ${ }^{1-7}$ Solitons are localized formations in nonlinear systems, ${ }^{8-15}$ appearing in many nonlinear processes, from fluid and biology dynamics, to plasma physics and fiber lasers, ${ }^{16-19}$ and especially in mode-locked lasers. ${ }^{5,11,20}$ The mode-locked fiber laser, as an ideal platform, is used for exploring nonlinear phenomena due to its compact and low-cost configuration as well as excellent features of high stability and low noise. Solitons observed in mode-locked lasers exhibit several special behaviors, such as soliton bunching and soliton bounding (i.e., the generation of soliton molecules). ${ }^{21-23}$

In the stationary state, the soliton train generated from mode-locked lasers can be described theoretically by means of the generalized nonlinear Schrödinger equation $(\mathrm{NSE})^{17}$ or the Ginzburg-Landau equation (GLE). ${ }^{11}$ Despite the ultimate

*Address all correspondence to Xueming Liu, E-mail: liuxueming72@yahoo.com stability of the mode-locked pulse train, its initial self-starting process contains a rich variety of unstable phenomena that are highly stochastic and nonrepetitive. ${ }^{24-28}$ The theoretical models of these dynamical processes are beyond the NSE and GLE. While conventional technologies cannot generally measure these rapid nonrepetitive processes due to the limited measurement bandwidth, ${ }^{1}$ the recently developed time-stretch dispersive Fourier transform (TS-DFT) technique can provide an elegant way to obtain real-time, single-shot measurements of ultrafast optical phenomena. ${ }^{29-34}$ This technique helped scientists to experimentally resolve the evolution of femtosecond soliton molecules, ${ }^{30}$ the internal motion of dissipative optical soliton molecules, ${ }^{17,35}$ and the dynamics of soliton explosions. ${ }^{36-38}$ Some successful examples of using this TS-DFT technique include the observations and measurements of rogue wave dynamics, modulation instability, and supercontinuum generation. ${ }^{28,32,33}$

The starting dynamics of passive mode-locking lasers have been established by a large set of experimental and theoretical investigations over the past two decades. ${ }^{39-48}$ Before the 
emerging TS-DFT technique, the transient dynamics in modelocked lasers had been investigated by using an oscilloscope. ${ }^{24,26}$ The real-time spectral evolution in the buildup process cannot be resolved, however, without the TS-DFT technique. ${ }^{1}$ Recently, both the transient spectral and temporal dynamics were observed with the assistance of the TS-DFT technique. ${ }^{34,35}$ Environmental perturbations (e.g., the polarization change in laser cavity and the fluctuation of pumping strength) can cause the lasers to sustain the extra unstable stages, such as $Q$-switched lasing. ${ }^{34,35}$ To reveal the true buildup process of solitons, we must mitigate the environmental perturbation as far as possible. This issue has not yet been overcome, unfortunately, although the self-starting process of mode-locked lasers was demonstrated in previous reports. ${ }^{24,26,34,35,49}$ Recently, Peng et al. ${ }^{50,51}$ reported the real-time observation of dissipative soliton formation and the buildup of dissipative optical soliton molecules. Relaxation oscillations were excluded from their reports, so they observed partial rather than total buildup processes. In our previous report, ${ }^{52}$ we observed the buildup process of soliton molecules rather than solitons, yet no theoretical model was proposed to describe the entire buildup process.

In the present study, $Q$-switched lasing is availably suppressed by decreasing the environmental perturbation and optimizing the laser system with the assistance of polarizationinsensitive carbon nanotube saturable absorber (CNT-SA). We therefore observe the entire buildup process of solitons in mode-locked lasers, for the first time to the best of our knowledge, in which there exist two possible pathways to generate the laser solitons. One pathway comprises the raised relaxation oscillation, quasimode-locking (Q-ML) stage, spectral beating dynamics, and stable mode-locking. The other pathway includes, however, an extra transient bound-state stage prior to the final mode-locking operation. The numerical simulations based on the roundtrip circulating-pulse method confirm the experimental observations. Moreover, a theoretical model is proposed to investigate the raised relaxation oscillation during the buildup process of laser pulses. The buildup time for the birth of solitons is predicted and agrees well with the experimental measurements. These findings highlight the importance of real-time measurements and provide some perspectives into the ultrafast transient dynamics of nonlinear systems.

\section{Experimental Configuration and Theoretical Model}

Due to the speed limitation of electronic devices, the real-time oscilloscope hardly resolves the ultrashort pulse with the duration of $<10 \mathrm{ps}$. However, the mode-locked lasers usually deliver ultrashort pulses (e.g., the pulse duration is $<1 \mathrm{ps}$ in the modelocked fiber lasers ${ }^{42}$ and even $20 \mathrm{fs}$ in the Kerr-lens mode-locked Ti:sapphire lasers $\left.{ }^{1}\right)$. The oscilloscope cannot resolve the soliton buildup process in mode-locked lasers if no special technique is used. An example is shown in Video 1 (Fig. 1).

Ultrashort pulses are linearly dispersed in the fiber, mapping their spectrum to the time domain,$^{53}$ according to the TS-DFT technique. ${ }^{1,30,54}$ As a result, the oscilloscope can resolve the real-time spectral evolution of the soliton buildup process. Two examples are shown in Videos 2 and 3 (Figs. 2 and 3). The measurement method is presented in Sec. 6 .

To suppress $Q$-switched lasing, the laser system has to be optimized due to the presence of temperature variation, mechanical vibration, and pumping strength fluctuation. We

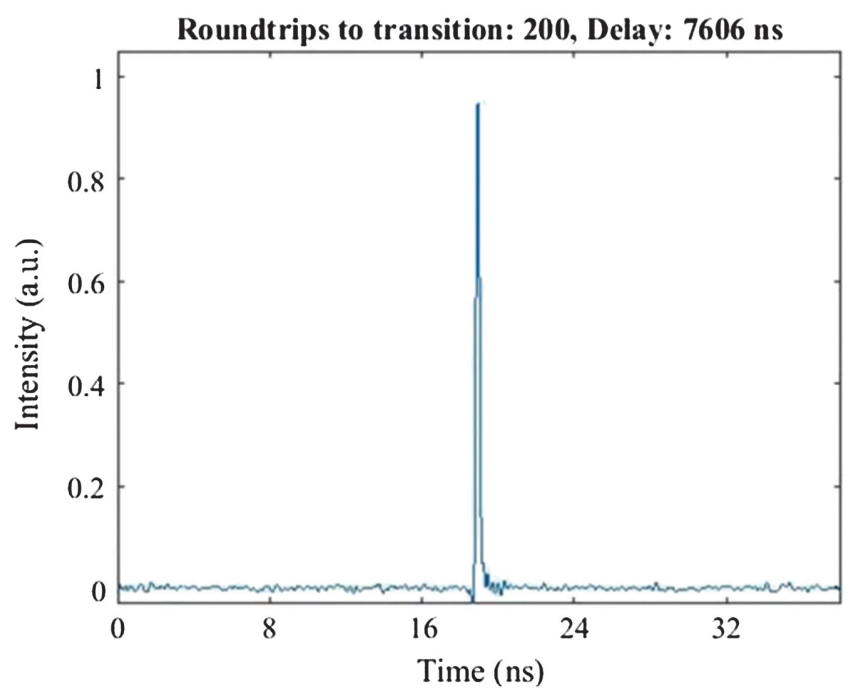

Fig. 1 Experimental real-time measurements from undispersed events (i.e., not using TS-DFT) for the formation and evolution of a soliton, where the beating phenomenon cannot be discovered [Video 1, MOV, 3.34 MB (URL: https://doi.org/10.1117/1.AP.1.1 $.016003 .1)]$.

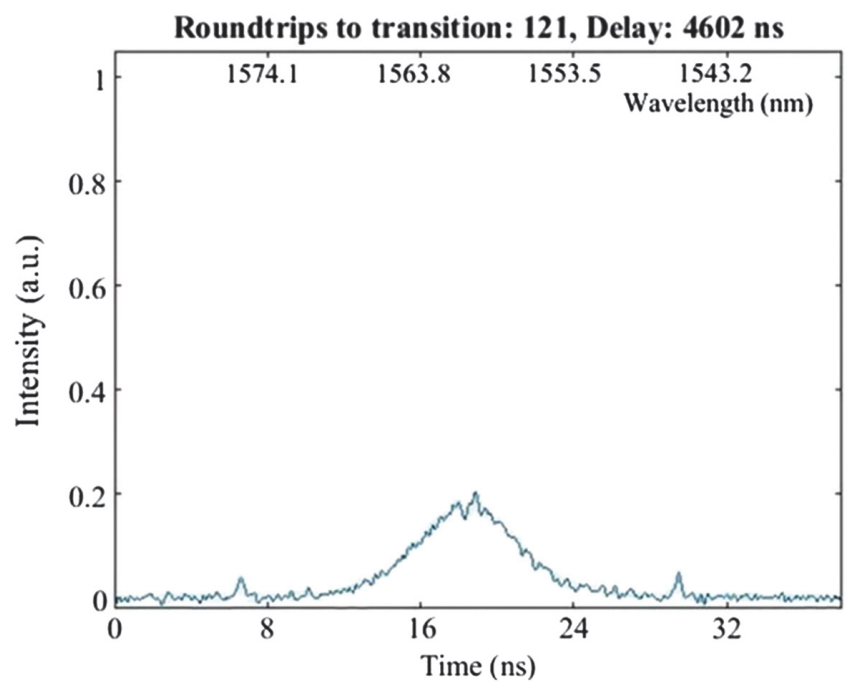

Fig. 2 Experimental real-time observation for the formation and evolution of a soliton, including Q-ML, beating dynamics, and stable mode-locking [Video 2, MOV, 3.69 MB (URL: https://doi .org/10.1117/1.AP.1.1.016003.2)].

use a compact hybrid combiner, which consists of a wavelength division multiplexer, a tap coupler, and an isolator. In addition, a polarization-insensitive saturable absorber based on carbon nanotubes is used as a mode-locking device. As a result, the stability of the laser system is greatly improved. The experimental setup is shown in detail in Sec. 6.

The numerical simulations for the entire buildup process of solitons in mode-locked lasers are quite important to revealing the underlying mechanism. However, the numerical methods have not been proposed so far. To solve this problem, we propose a two-step method that can theoretically describe the entire buildup process of mode-locked lasers, as shown in Secs. 7 and 


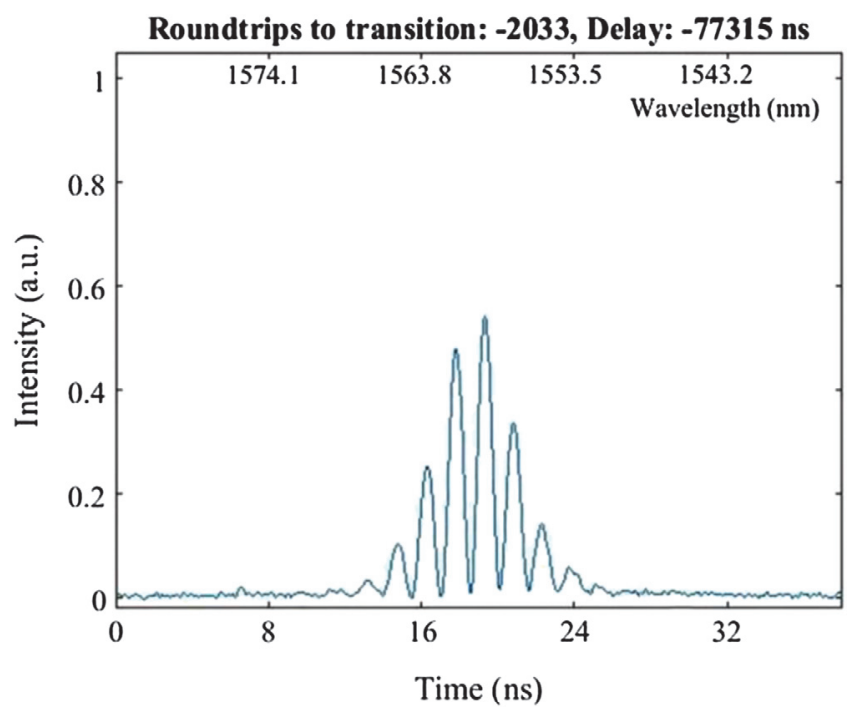

Fig. 3 Experimental real-time observation for the formation and evolution of a soliton, including Q-ML, beating dynamics, transient bound state, and stable mode-locking [Video 3, MOV, 6.99 MB (URL: https://doi.org/10.1117/1.AP.1.1.016003.3)].

8. In the first step, the model proposed in Sec. 7 is used to simulate the raised relaxation oscillation process and predict the buildup time and nascent time. In this model, the total optical field in the laser cavity is the superposition of multiple modes. In the second step, the extended NSE and roundtrip circulatingpulse method (see Sec. 8) are used to find the two different evolution processes of soliton buildup and simulate the Kelly sidebands of solitons.

\section{Experimental Results}

\subsection{Buildup Dynamics of Solitons with Beating Dynamics}

The time-stretch technique can overcome the speed limitation of electronic digitizers. ${ }^{54}$ The spectral interferograms are mapped into the time domain by using the TS-DFT technique. The timing data are measured via direct detection [see Fig. 4(a)], whereas the real-time spectra are obtained by dispersing the laser pulses over a 5-km length of dispersion-compensating fiber (DCF) prior to detection [see Fig. 4(b)]. The pulse-resolved acquisition and real-time spectral acquisition are measured simultaneously (see experimental setup in Sec. 6). Compared to the direct detection data of the laser output, the real-time spectral data are delayed in time by $\sim 24.5 \mu$ s due to the additional 5-km DCF.

The direct measurement and TS-DFT data are plotted in Figs. 4(a) and 4(b), respectively. While the direct detection gives a temporal trace of the laser output as shown in panel (A), the TS-DFT technique provides the single-shot measurement of the laser spectral information as shown in panel (B). The experimental observations reveal that there exists an evidently raised relaxation oscillation before the appearance of a stable pulse train (i.e., stable mode-locking). A representative real-time measurement is exhibited in Fig. 4(b), where the duration of the raised relaxation oscillation is $\sim 4.6 \mathrm{~ms}$, corresponding to $\sim 1.2 \times 10^{5}$ cavity roundtrips. Before $4.32 \mathrm{~ms}$, the number of cavity photons remains at the initial low value, as determined by quantum field fluctuations. ${ }^{55}$ From this time on, the first laser spike is generated. The separation of the neighboring laser spikes is $\sim 80 \mu \mathrm{s}$. The buildup time of solitons from the beginning time of pumping process to the stable mode-locking is $\sim 4.65 \mathrm{~ms}$. Note that, in the experiments, we only need to measure the buildup period from the first laser spike to the stable mode-locking, and, thus, we define the duration of this process as the nascent time of the laser (here the nascent time is $\sim 0.35 \mathrm{~ms}$ ).

Usually, the buildup process of mode-locked lasers includes the raised relaxation oscillation, Q-ML stage, beating dynamics, and finally the stable mode-locking state, as shown in Fig. 4. The experimental results show that the laser spikes are generated gradually. Figure 4(c) shows the expanded views of the fifth laser spike in Fig. 4(b). Figure 4(c) shows that, prior to the stable mode-locking, there exist a Q-ML and beating dynamics with the duration of $\sim 30 \mu \mathrm{s}$. During the Q-ML stage, multiple subordinate pulses appear together with a dominant pulse as shown in panel (C). Panel (B) reveals that the TS-DFT technique can achieve spectral information rather than temporal information on ultrashort time scale. But, the direct detection without the TS-DFT technique is used to obtain the temporal information, as shown in panel (A). The panels (A) to (C) show that the roundtrip time of laser is $38.03 \mathrm{~ns}$.

The time-continuous data stream shown in Fig. 4(b) evolves with a periodicity of $38.03 \mathrm{~ns}$, which corresponds to the cavity roundtrip time. We therefore segment the time series into intervals of roundtrip time (i.e., $38.03 \mathrm{~ns}$ ), achieving a twodimensional (2-D) (matrix) representation exhibited in Fig. 5(a). Here, the vertical and horizontal axes depict the information within a single roundtrip and the dynamics across consecutive roundtrips, respectively. The 2-D representation (also named as spatiotemporal representation) was proposed to describe the nonlinear system with delayed feedback; ${ }^{56}$ analogous techniques were used in several optical systems, such as the mode-locked fiber laser, ${ }^{57}$ cavity soliton in fiber-ring resonator, ${ }^{58}$ and injection-locked semiconductor laser. ${ }^{59}$

Figure 5(a) exhibits the complex formation process of the pulse laser, involving Q-ML stage, beating dynamics, and ultimately the stationary single-soliton mode-locking state. A video illustrates the experimental real-time observation in detail in Fig. 2. There exist multiple pulses in the laser cavity during the Q-ML stage and the beating dynamics, whereas only a dominant pulse gradually evolves to the final stationary mode-locking pulse. The last frame in Fig. 5(a) is illustrated in Fig. 5(b). The corresponding optical spectrum, demonstrated in Fig. 5(c), is measured directly by an optical spectrum analyzer (OSA). Clear Kelly sidebands are observed in Fig. 5(c), being the typical characteristics of soliton fiber lasers. ${ }^{60-63}$ The full-widthat-half-maximum spectral width of the solitons is $\sim 8.2 \mathrm{~nm}$. Figure 5(d) provides a close-up of the data from Fig. 5(a), revealing the beating dynamics with an interference pattern. Experimental real-time measurements from undispersed events (i.e., not using TS-DFT technique) are demonstrated in Fig. 5(e), where the beating phenomenon cannot be discovered (see the animation in Video 1).

\subsection{Buildup Dynamics of Solitons with Transient Bound State}

Figures 4 and 5 show that the beating dynamics occurs prior to the stable mode-locking. Some of our experimental observations 


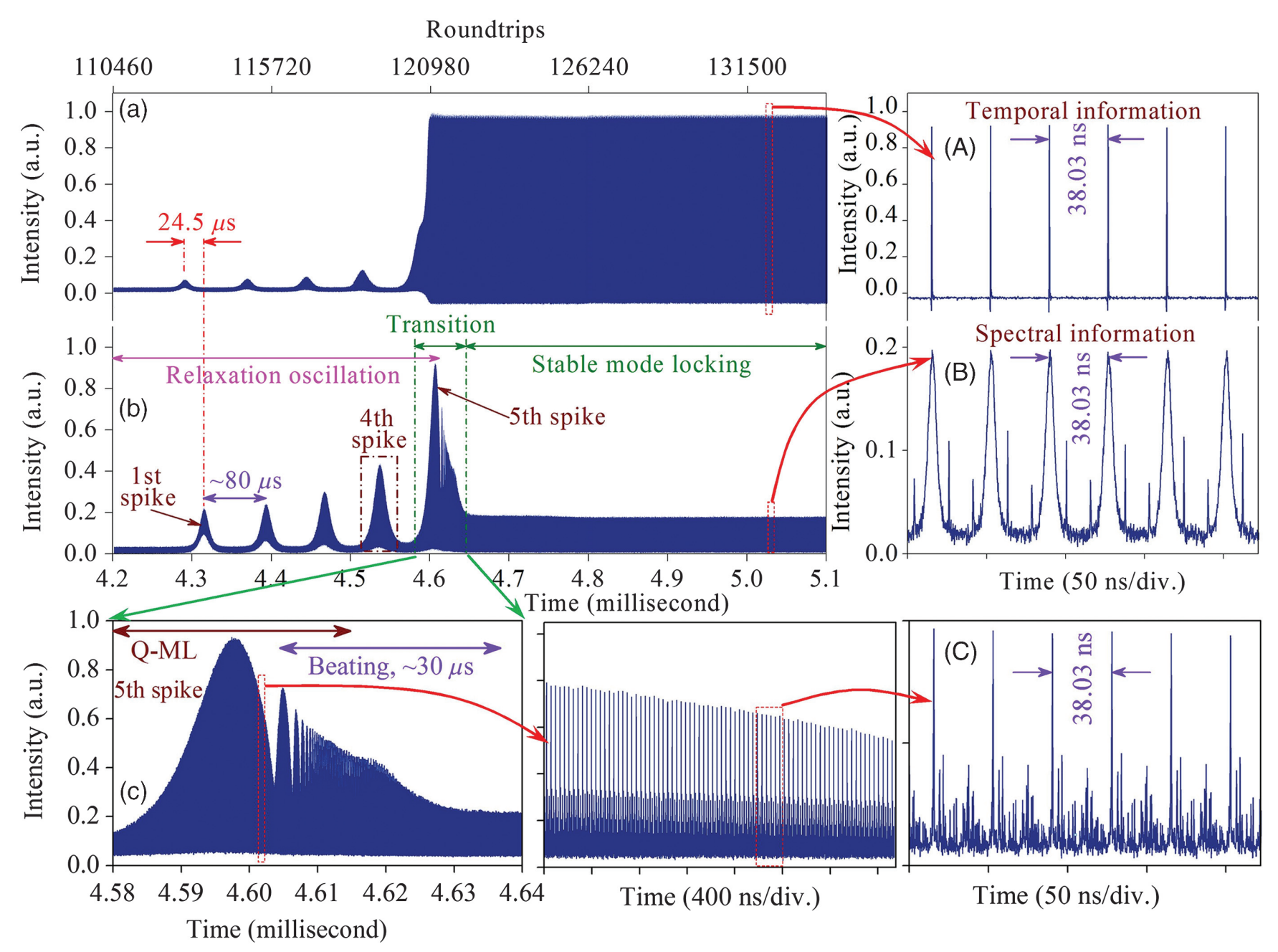

Fig. 4 Experimental real-time display of the buildup dynamics of solitons in mode-locked laser, captured simultaneously by using (a) an undispersed and (b) a dispersed element, respectively. (a) Direct detection with a high-speed photodetector and a real-time oscilloscope. (A) The expanded view shows that the timing data represent the temporal information. (b) The buildup dynamics are obtained by dispersing the solitons in 5-km DCF prior to detection, as denotes the single-shot spectral information shown in (B). The timing data advance the real-time spectral data about $24.5 \mu \mathrm{s}$ that is delayed by 5-km DCF. (c) Close-up of the data from (b) for the fifth laser spike, revealing the quasi mode-locking (Q-ML) and beating dynamics with the duration of $\sim 30 \mu \mathrm{s}$. (C) There are multiple subordinate pulses together with a dominant pulse in the laser cavity. The period for $(A)$ to $(C)$ is $38.03 \mathrm{~ns}$, corresponding to the roundtrip time of the laser cavity. The buildup process of mode-locked lasers includes the raised relaxation oscillation, Q-ML stage, beating dynamics, and stable mode-locking.

demonstrate, however, that a transient bound state of two solitons may appear between the two stages. A typical example is illustrated in Fig. 6, and the corresponding animation is included in Video 3. The real-time TS-DFT measurement of this unique buildup process, shown entirely in Fig. 6(a), includes the raised relaxation oscillation, the transition region, and the final stationary single-pulse mode-locking state. The unique transition region shown in Fig. 6(a) has a duration $\sim 3$ times longer than that in Fig. 4(b). Figure 6(b), which is quite similar to panel (B) in Fig. 4, is the expanded view at the stable mode-locking in Fig. 6(a). Figure 6(c) is a 2-D representation of some data from Fig. 6(a), which shows the evolution of an optical wave during this transition region in detail. The interferograms show the periodical modulation along with wavelength, which is the typical result of bound-state spectrum. ${ }^{17,30}$ Figure 6(e) demonstrates the Fourier transform of each single-shot spectrum of the transient bound state. Obviously, the corresponding field autocorrelation with three peaks exhibits the evolution of bound state with two pulses. ${ }^{30}$

We set the beginning time of the stationary single-pulse mode-locking state as 0 roundtrip number for convenience of reference, as shown in Fig. 6(c). For example, the roundtrip number of -800 corresponds to the recording time of 800 roundtrips prior to the stable mode-locking state. Figure 6(c) shows that the two solitons of transient bound state are generated via the beating effect at a roundtrip of about -2800 , where 


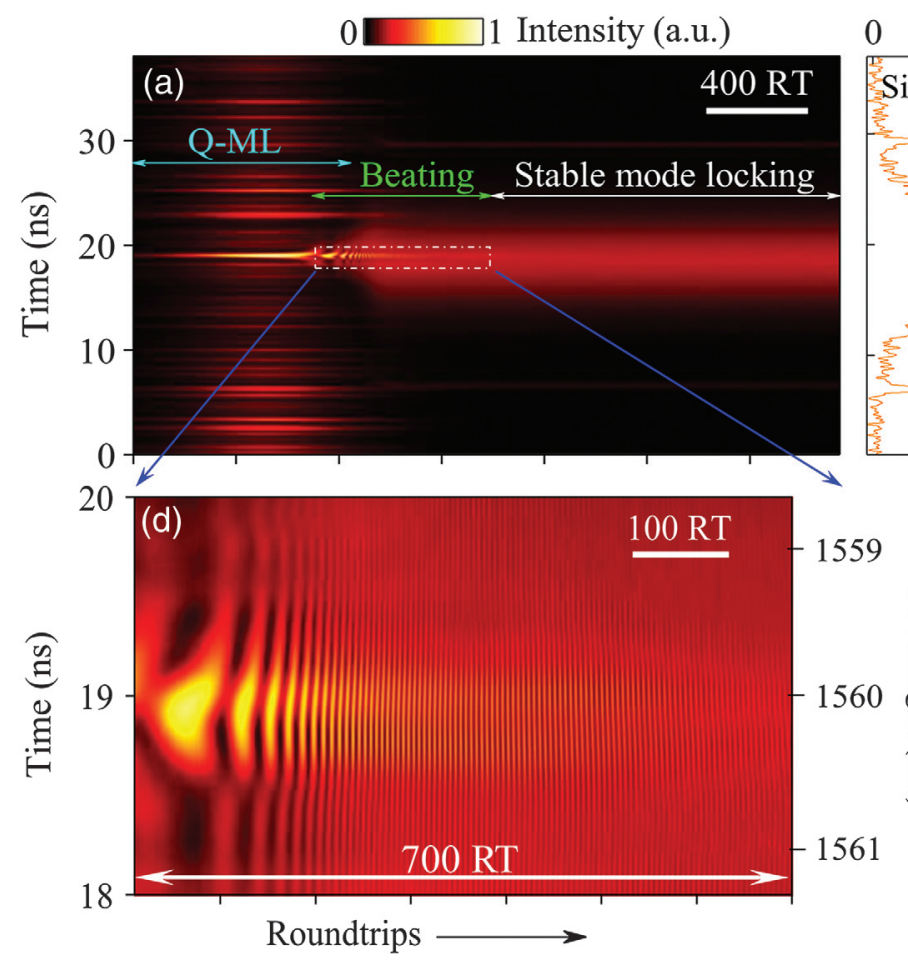

Intensity (a.u.)

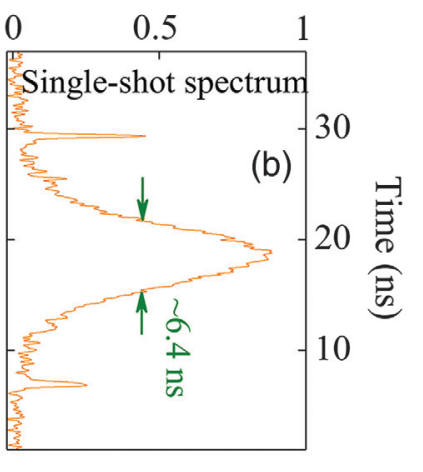

Intensity (a.u.)

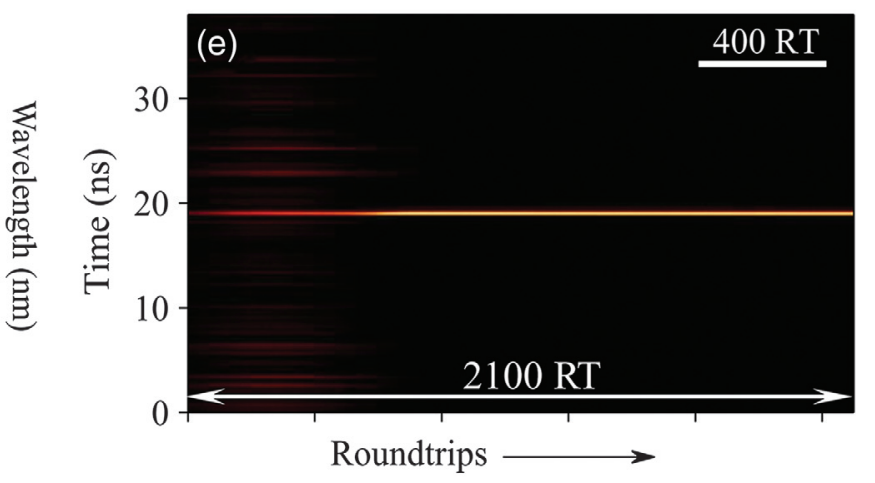

Fig. 5 Formation of a soliton with beating dynamics. The recorded time series is segmented with respect to the roundtrip time and displays the buildup dynamics of a soliton. The intensity profile evolves along with time (vertical axis) and roundtrips (horizontal axis). (a) Experimental real-time observation during the formation of a soliton from the Q-ML and beating behavior to the stable mode-locking (see Video 2 for the full animation). The experimental data are from Fig. 4(b). TS-DFT maps the spectral information into the temporal domain. (b) Exemplary singleshot spectrum, corresponding to the last frame in (a). (c) Optical spectrum of soliton measured by an OSA. (d) Close-up of the data from (a), revealing the interference pattern for the beating dynamics. (e) Experimental real-time observation via direct measurement (see Video 1 for the full animation), without using TS-DFT technique. The beating dynamics are not revealed in the direct measurement. [RT: roundtrip (scale bar).]

the separation of solitons is zero [Fig. 6(e)]. During the transition, the two solitons evolve in a rather complex manner, and finally the bound state collapses due to the increasing separation between them. The single-shot spectrum at the steady state is similar to the results presented in Fig. 5(b). The relative phase, $\alpha$, between the two solitons experiences eight abrupt changes during the roundtrips from -1840 to -710 [see Fig. 6(f)], which are revealed by the eight circles in the interaction plane shown in Fig. 6(d). The relative phase evolves on the opposite direction after the roundtrip number of about -1100 [Fig. 6(f)] so that the rotating direction reverses after this inflection point [Fig. 6(d)].

Figure 6(c) exhibits the spectral interference since the TSDFT technique is used to obtain spectral information rather than temporal information. The Fourier transform of each single-shot spectrum only achieves the field autocorrelation of the momentary bound state, ${ }^{30}$ as shown in Fig. 6(e). To extract the timing information of transient bound states, we assume a bound state as a superposition of temporally separated individual solitons. The bound state field can be expressed as ${ }^{17,30}$

$E(t)=\left[E_{1}(t)+E_{2}(t) \exp (i \alpha)\right] \exp \left(i \omega_{0} t\right)$.
Here, $\omega_{0}$ is the common carrier frequency. The temporal solitons usually have the hyperbolic secant envelopes, ${ }^{42,63,64}$ i.e.,

$E_{1}(t)=a_{1} \operatorname{sech}\left[(t-R / 2) / \Delta t_{1}\right]$

$E_{2}(t)=a_{2} \operatorname{sech}\left[(t+R / 2) / \Delta t_{2}\right]$

where $a_{1}$ and $a_{2}$ are the amplitudes of the two solitons in the transient bound state, respectively, and $\Delta t_{1}$ and $\Delta t_{2}$ are the corresponding pulse widths. After the manipulation of Fourier transform, the spectral intensity $I(\Omega)$ of the optical field is given as

$$
\begin{aligned}
I(\Omega)= & A_{1}^{2} \operatorname{sech}^{2}\left(\frac{\pi}{2} \Delta t_{1} \Omega\right)+A_{2}^{2} \operatorname{sech}^{2}\left(\frac{\pi}{2} \Delta t_{2} \Omega\right) \\
& +2 A_{1} A_{2} \operatorname{sech}\left(\frac{\pi}{2} \Delta t_{1} \Omega\right) \operatorname{sech}\left(\frac{\pi}{2} \Delta t_{2} \Omega\right) \cos (\Omega R+\alpha),
\end{aligned}
$$

where $A_{1}=\sqrt{\pi / 2} a_{1} \Delta t_{1}, A_{2}=\sqrt{\pi / 2} a_{2} \Delta t_{2}$, and $\Omega=\omega-\omega_{0}$. 

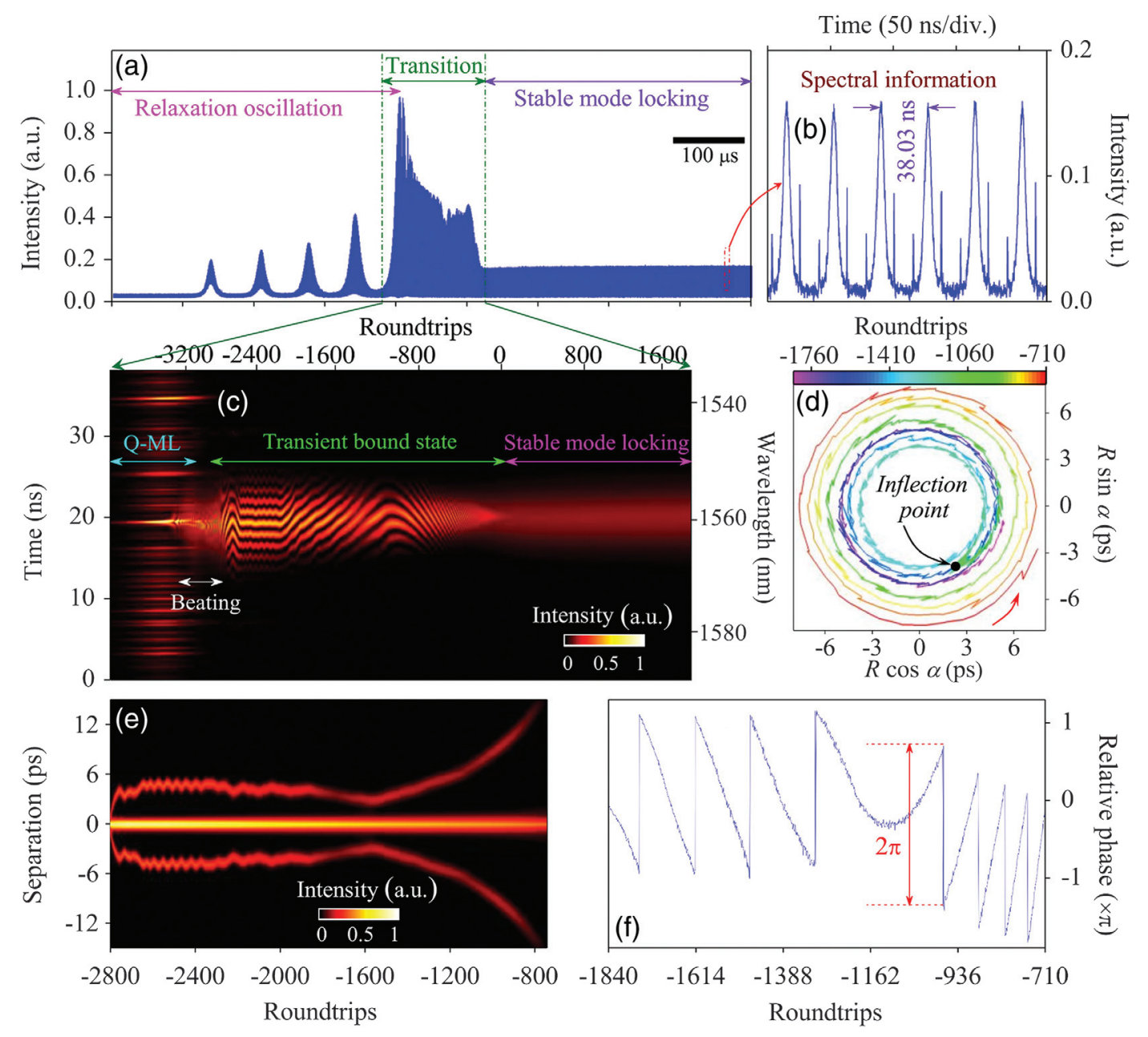

Fig. 6 Experimental real-time display of the buildup dynamics of solitons with transient bound state. (a) Buildup dynamics via the TS-DFT technique. The entire buildup process includes the raised relaxation oscillation, transition region, and stable mode-locking. (b) Close-up of the data from (a) at the stable mode-locking state, as denotes the single-shot spectral information. (c) Experimental real-time interferogram during the formation of a soliton, accessed via the dynamics of Q-ML, beating dynamics, transient bound state, and stable mode-locking. For a full animation of the observations, see Video 3. For convenience of reference, the beginning time of stable mode-locking is set to zero roundtrips. (d) Dynamics of the solitons mapped in the interaction plane over 1000 roundtrips. The angle, $\alpha$, represents the relative phase of two solitons. The radius, $R$, corresponds to the bound-state separation. Two solitons gradually depart from each other. (e) The Fourier transform of each single-shot spectrum corresponds to a field autocorrelation of the momentary bound state, tracing the separation between both solitons.

(f) The relative phase between both solitons along with roundtrips.

On the basis of the experimental data shown in Fig. 6, $a_{1}, a_{2}$, $\Delta t_{1}$, and $\Delta t_{2}$ can be achieved by solving Eq. (4) with a nonlinear least-square solver. Then, the temporal intensity, $|E(t)|^{2}$, for the bound state field is shown in Fig. 7. It can be seen that the two solitons in the transient bound state have different amplitudes and pulse widths. The two solitons are generated quickly from the roundtrip of around -2800 , and then their separation fluctuates within a range of $\sim 5.5$ ps before the roundtrip number of about -1250 . In succession, the two solitons start to depart from each other. Finally, one of the solitons disappears, leaving the other one evolving to the stationary mode-locking pulse inside the laser cavity. The system ultimately settles on the stable mode-locking with only one soliton.

\section{Discussions}

\subsection{Buildup Process of Mode-Locked Lasers with Q-Switched Lasing}

Kerr-lens mode-locked laser is able to deliver the pulses and solitons, ${ }^{1,30}$ in which the nonlinear effect plays a critical role. To simplify the system and improve the stability of lasers, the mode-locked fiber lasers have been proposed on the basis of the nonlinear polarization rotation (NPR) technique. ${ }^{34,49}$ However, the experimental observations showed that the selfstarting process of mode-locked laser was quite sensitive to environmental perturbations, such as the instability of laser 


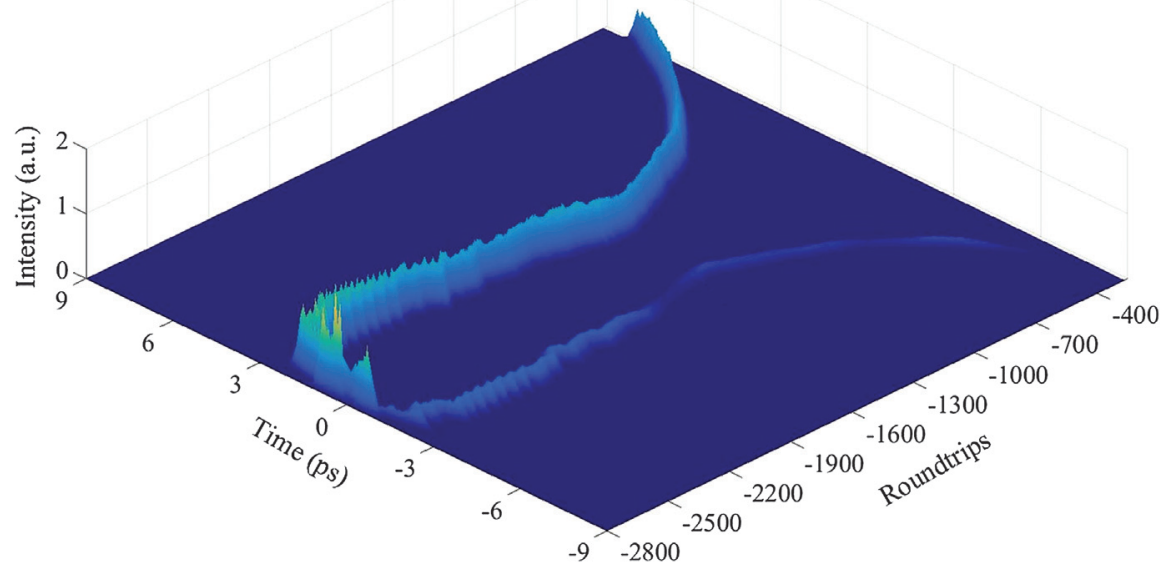

Fig. 7 Interaction and evolution of two solitons in the transient bound state. The temporal solitons are extracted from the experimental data shown in Fig. 6 by using the Levenberg-Marquardt algorithm. The stronger soliton gradually evolves to the stationary state; however, the weaker one ultimately vanishes via the complex dynamics. The roundtrips are from about -2800 to -300 , and the corresponding autocorrelation intensity of the momentary bound state is shown in Fig. 6(e).

diode (LD), the polarization change in laser cavity, and the fluctuation of pumping strength. Therefore, the change of polarization state in lasers can extend the buildup time of mode-locked lasers. For instance, the buildup time of lasers was $>10 \mathrm{~ms}^{1}$ and, even, $>100 \mathrm{~ms} .{ }^{35}$ Similar results are achieved in our works, as shown in Fig. 8.

To compare the experimental observations shown in Figs. 4, 5 , and 6, we establish a fiber laser mode-locked by NPR technique, in which the polarization change and the fluctuation of pumping strength can evidently influence the self-starting process. Figure 8(c) shows a typical buildup process of modelocked laser. Obviously, the $Q$-switched lasing occurs prior to the stable mode-locking. The number of $Q$-switched lasing is 189 with the duration of $>160 \mathrm{~ms}$. This result is very similar to the experimental observation reported in Ref. 35.

We improve the stability of pumping strength and decrease the environmental perturbation. The buildup time of laser was shortened to be about $80 \mathrm{~ms}$ with 76 lines of $Q$-switched lasing, as shown in Fig. 8(b). We further enhance the robustness of mode-locked laser by using the hybrid saturable absorber, where the single-wall carbon nanotubes, together with NPR technique, operate on the laser. The experimental result is demonstrated in Fig. 8(a), where the duration of $Q$-switched lasing is $\sim 3 \mathrm{~ms}$ and its number is 5 . Every $Q$-switched lasing is composed of several hundreds of pulses, as shown in the insets of Fig. 8(a).

By comparing Fig. 8(a) to Figs. 8(b) and 8(c), it can be seen that the decrease of environmental perturbation can effectively shorten the buildup time of laser and suppress the $Q$-switched lasing. Figure 8(a) shows that, therefore, the laser experiences a shorter unstable $Q$-switched lasing stage. Note that, within Fig. 8(a), the separation between the neighboring $Q$-switched lasing lines is $\sim 700 \mu \mathrm{s}$. However, the separation between the laser spikes in the raised relaxation oscillation is $\sim 80 \mu \mathrm{s}$, as shown in Fig. 4(b).

When the polarization-dependent devices are removed from the laser cavity, only single-wall carbon nanotube serves as the saturable absorber. Simultaneously, we optimize the laser system and the saturable absorber. As a result, the $Q$-switched lasing is suppressed completely, and then no $Q$-switched lasing occurs in the self-starting process of mode-locked laser. The raised relaxation oscillation is observed experimentally, as shown in Fig. 9(a) below. Therefore, the experimental results here denote the general buildup process, which can reflect the intrinsic features of mode-locked lasers.

\subsection{Buildup Process of Mode-Locked Lasers without Q-Switched Lasing}

Figure 8 shows that the $Q$-switched lasing can cover up the true buildup dynamics of mode-locked lasers. The polarizationindependent mode locker (e.g., single-wall carbon nanotubes) can availably suppress the $Q$-switched lasing after the laser system is optimized. A typical result for the buildup process is demonstrated in Fig. 9. Figures 9(a) and 9(b) show the experimental results and the theoretical predictions, respectively. The red and blue curves denote the evolution of the pumping rate and raised relaxation oscillation along with time, respectively. The theoretical model for describing the raised relaxation oscillation is shown in Sec. 7.

The relaxation oscillation is a general transient behavior of laser. ${ }^{55}$ The laser without a mode locker undergoes a damped oscillatory behavior and, finally, approaches to the stable continuous-wave $(\mathrm{CW})$ operation. ${ }^{65}$ In contrast, the mode-lockerbased laser experiences a raised relaxation oscillation rather than a damped behavior, as shown in Fig. 9. Such relaxation oscillation was not observed in previous reports, ${ }^{24,26,34,35,49}$ since the $Q$-switched lasing leads the laser to depart from such phenomena. The mode locker can drive many longitudinal modes from randomized to synchronized, where the phase difference $\theta$ between any two neighboring modes is gradually locked to a constant value. During the raised relaxation oscillation, $\theta$ is a variable rather than a constant. We therefore 


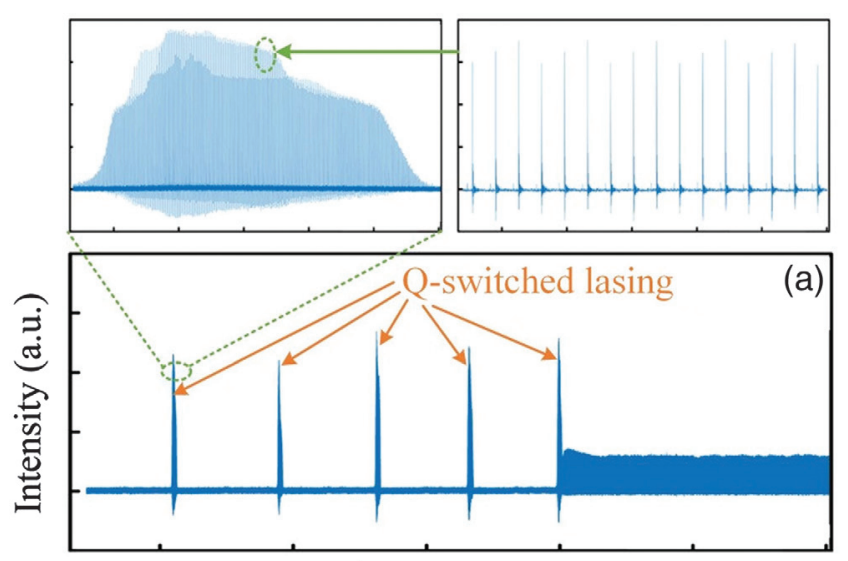

Time (1 ms/div.)
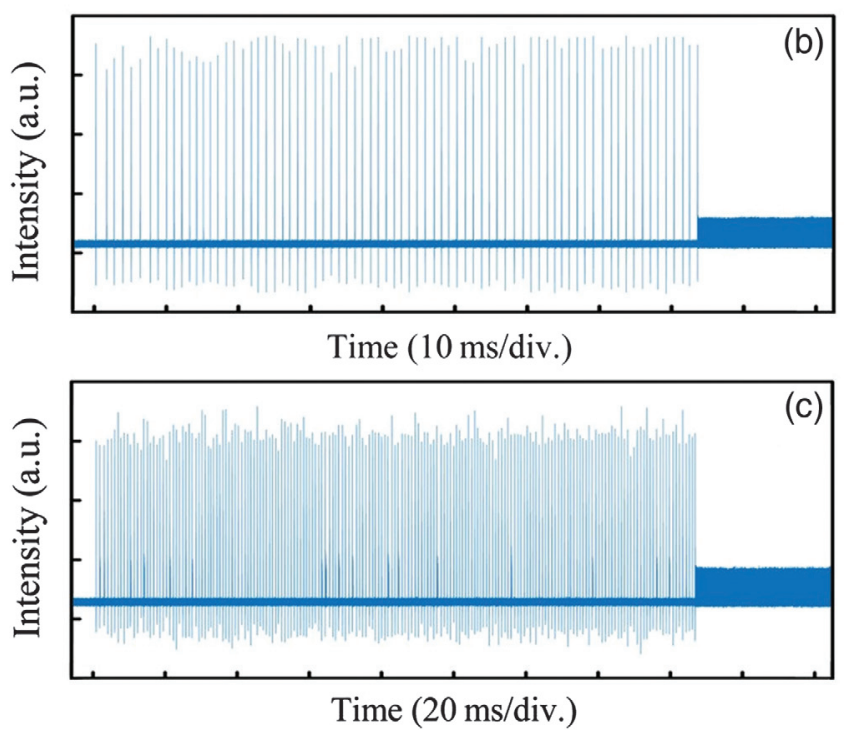

Fig. 8 Experimental buildup process of mode-locked laser with $Q$-switched lasing. The laser experiences an unstable $Q$ switched lasing stage during the buildup process. (a) Hybrid saturable absorber based on the single-wall carbon nanotubes together with NPR technique. The duration of $Q$-switched lasing is about $3 \mathrm{~ms}$ with five lines. The separation between the neighboring $Q$-switched lasing lines is $\sim 700 \mu \mathrm{s}$. Inset (left): close-up of the data from (a) for a $Q$-switched lasing. Inset (right): expanded view of a part from the left inset figure. (b) NPR-based saturable absorber together with weaker fluctuation of pumping strength. The duration of $Q$-switched lasing is $>80 \mathrm{~ms}$ with 76 lines. (c) NPR-based saturable absorber together with stronger fluctuation of pumping strength. The duration of $Q$-switched lasing is $>160$ ms with 189 lines.

define the former (i.e., a variable for $\theta$ ) as Q-ML and the latter (i.e., a constant for $\theta$ ) as perfect or stable mode-locking. The transition stage from Q-ML to stable mode-locking undergoes a beating dynamic [see Fig. 5(a)], which is described as auxiliary-pulse mode-locking by Herink et al. ${ }^{1}$ Moreover, both beating and transient bound state stages occur at the transition from Q-ML to stable mode-locking (see Fig. 6), which are observed here for the first time.

The buildup time is defined as the duration from the beginning time (i.e., turn-on time) of pumping process to the stable mode-locking. The nascent time is defined as the duration from
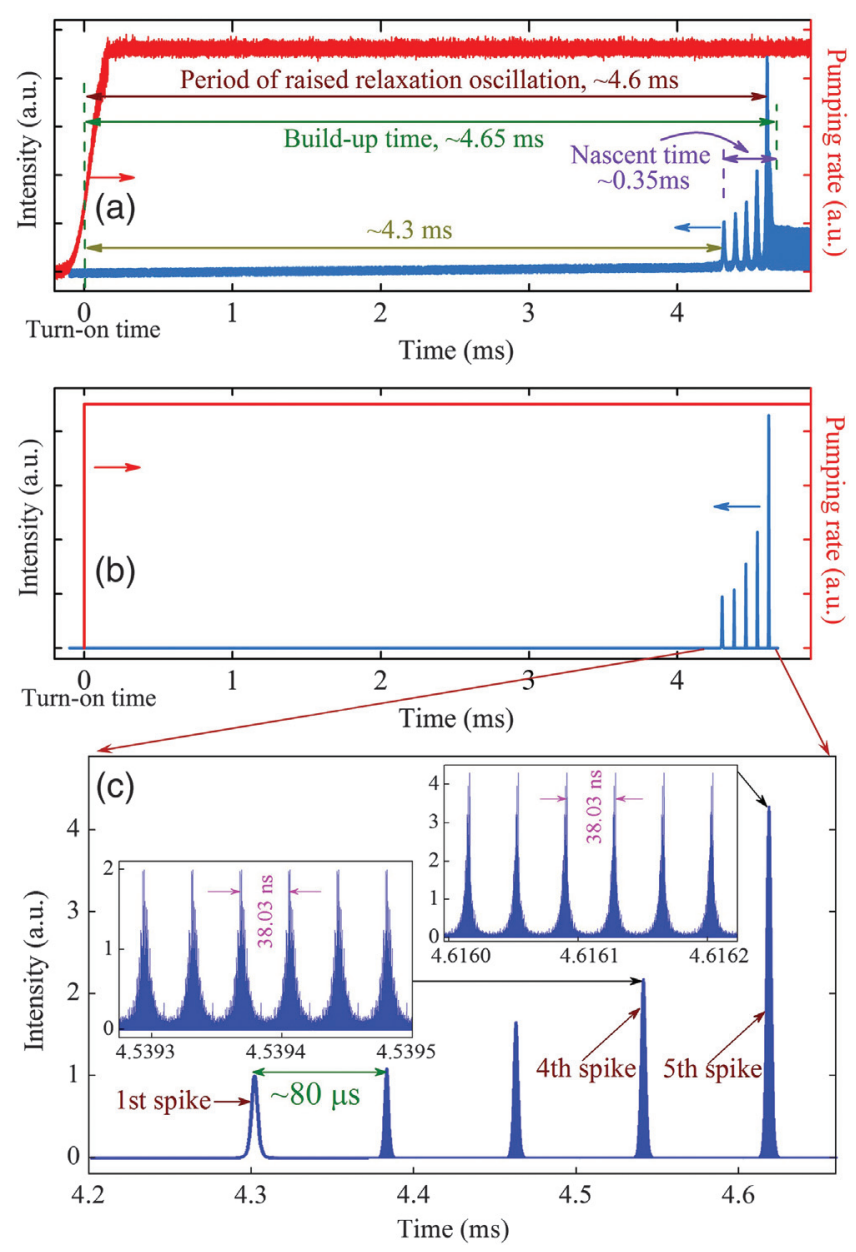

Fig. 9 Buildup process of mode-locked laser without $Q$-switched lasing. (a) Experimental results. (b) Numerical simulation, corresponding to (a). To numerically simulate the raised relaxation oscillation, we assume that the phase difference $\theta$ between any two neighboring modes is uniformly distributed from -4.8 to 4.8 , from -4.2 to 4.2 , from -3.65 to 3.65 , and from -2.86 to 2.86 for the second to fifth laser spikes, respectively. Red curves indicate the evolution of pumping rate along with time. Blue curves show the evolution of raised relaxation oscillation. (c) Close-up of (b). Two insets in (c) are the expanded view of the fourth and fifth laser spikes. Note that a.u. denotes arbitrary unit. The raised relaxation oscillation at the beginning stage of the mode-locking can be precisely predicted with several key features, matching excellently with the experimental measurements.

the first laser spike to the stable mode-locking. The period of raised relaxation oscillation is defined as the duration from the turn-on time to the last laser spike. Therefore, the buildup time and nascent time are $\sim 4.65$ and $0.35 \mathrm{~ms}$, respectively, as shown in Fig. 9(a). The first laser spike occurs at $\sim 4.3 \mathrm{~ms}$, and the period of raised relaxation oscillation is $\sim 4.6 \mathrm{~ms}$. Both the theoretical prediction and experimental observation show that, although the pump power is inputted into the laser system after the turn-on time, the number of cavity photons remains at the initial low value before the first laser spike (i.e., $\sim 4.3 \mathrm{~ms}$ ). Therefore, no pulse appears before $\sim 4.3 \mathrm{~ms}$, as shown in Fig. 9. 


\subsection{Theoretical Confirmation}

The proposed model in Sec. 7 successfully simulates the relaxation oscillation process, but it cannot describe the dynamics and evolution of pulses in the stationary mode-locking. Based on the extended NSE and the roundtrip circulating-pulse method (see Sec. 8), we can numerically achieve the two different evolutionary processes of soliton buildup. Figure $10 \mathrm{dem}-$ onstrates two typical results of numerical simulations, revealing two different evolutionary pathways of the soliton buildup. In one pathway, the single-soliton operation is directly formed via the beating dynamics, as shown in Figs. 10(a) and 10(b). Figures 10(a) and 10(c) exhibit the spectral and temporal evolutions of pulses, respectively. The simulation starts at an initial signal with the noise background that is illustrated as the red curve in Fig. 10(g). Obviously, the numerical results are in good agreement with the experimental observations shown in Fig. 5. When the simulation starts with another noise background field [see the black curve in Fig. 10(g)], the transient bound state can be observed in the buildup process, as shown in Fig. 10(d) (i.e., spectral evolution) and Fig. 10(f) (i.e., temporal evolution). In this way, the transient bound state with two solitons is generated at the beginning, however, the stronger soliton gradually evolves to the stationary state and the weaker one slowly decays and ultimately vanishes via the complex dynamics. The temporal evolution of two pulses in the numerical simulation [i.e., Fig. 10(f)] is quite similar to the experimental result (i.e., Fig. 7). Figures 10(b) and 10(e) present the close-ups of the white dotted-line boxes in Figs. 10(a) and 10(d), respectively. However, the finally stable solutions for two buildup pathways have the same values, as shown in Fig. 10(h) (i.e., spectral domain) and Fig. 10(i) (i.e., temporal domain). It should be noted that the two buildup pathways are achieved at the same laser parameters except that two initial noises [see Fig. 10(g)] have a slight difference.

The roundtrip circulating-pulse method together with NSE (see Sec. 8) can be used to simulate the two different evolution processes of soliton buildup and achieve the Kelly sidebands numerically, as shown in Fig. 10. However, they cannot simulate the raised relaxation oscillation at the beginning of the birth of
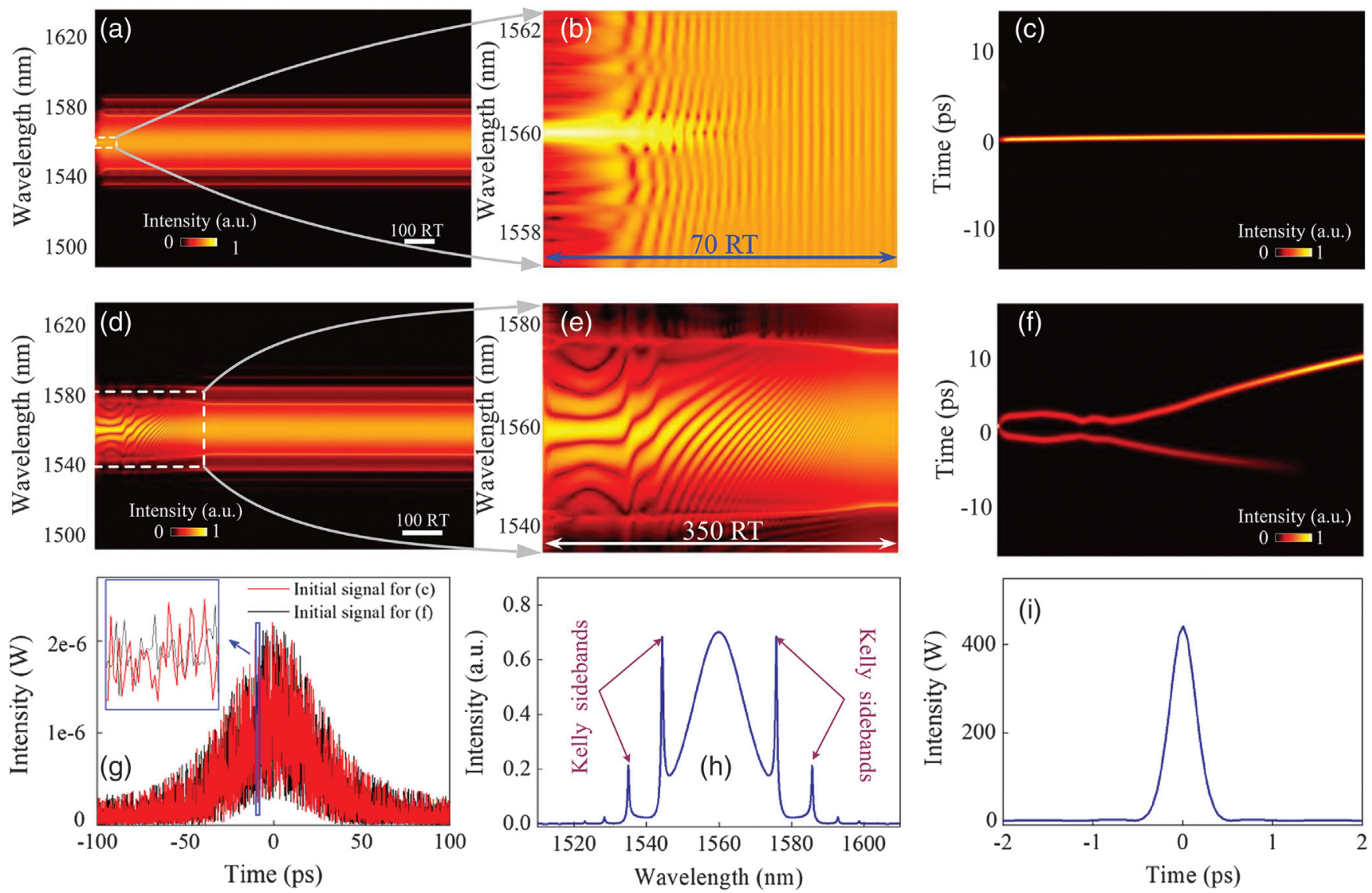

Fig. 10 Numerical simulations based on the roundtrip circulating-pulse method. (a)-(c) Soliton buildup process with beating dynamics in one pathway. (d)-(f) Soliton buildup process with transient bound state in the other pathway. (a) and (d) Spectral evolution of soliton along with roundtrips. (c) and (f) Temporal evolution of soliton along with roundtrips. (b) and (e) Close-ups of the data from the white dotted-line boxes in (a) and (c), respectively. (g) Initial signals with the noise background. The intensity of initial signals is in the order of magnitude of $10^{-6} \mathrm{~W}$, which is eight orders of magnitude lower than the intensity of stable solitons (i.e., $10^{2} \mathrm{~W}$ ). Inset: close-up of the data from the blue box. (h) Spectral and (i) temporal profiles at the finally stationary solution. Although the solitons experience through two different buildup pathways, they evolve to the same steady soliton solution with Kelly sidebands. 
solitons. Detailed description and analysis are given in Sec. 9. On the other hand, the proposed model in Sec. 7 can simulate the raised relaxation oscillation process and predict the buildup time and the nascent time to be $\sim 4.65$ and $0.35 \mathrm{~ms}$ [see Fig. 9(b)], respectively. But it cannot achieve the dynamics and evolution of solitons (e.g., Fig. 10). Here, by combining Secs. 7 and 8, we have proposed a two-step method to simulate the entire buildup process of solitons in mode-locked lasers, including the raised relaxation oscillation, transient bound state, Kelly sidebands, and beating pattern.

\section{Conclusions}

The $Q$-switched lasing covers up some important phenomena in the buildup process of mode-locked lasers and results in a lengthy self-starting time (see Fig. 8). By optimizing the laser system and the mode locker, we completely suppress the $Q$ switched lasing that is induced by environmental perturbation. The experimental results show that, without the $Q$-switched lasing, the buildup time of mode-locked lasers is $\sim 4.65 \mathrm{~ms}$ and the nascent time of solitons is $\sim 0.35 \mathrm{~ms}$ [see Figs. 4(b) and 9(a)]. We experimentally observed the real-time spectral dynamics of the entire buildup process of solitons for the first time, to the best of our knowledge, by using the TS-DFT technique. It was discovered that there are two pathways for the buildup process of solitons in mode-locked lasers. The entire buildup process usually includes the raised relaxation oscillation, $Q$ ML stage, and beating dynamics in one pathway (see Fig. 4) and even contains extra dynamics, such as the transient bound state of two solitons, in another pathway (see Fig. 6). We have proposed a theoretical model to describe the raised relaxation oscillation in the birth of solitons (see Sec. 7), which can successfully predict the buildup time of solitons (see Fig. 9). Based on this model together with the extended NSE and roundtrip circulating-pulse method (see Sec. 8), we have proposed a two-step method that can accurately simulate the entire soliton buildup process. These findings provide insights into the ultrafast transient dynamics of mode-locked lasers, and bring perspectives useful for the context of laser design and applications. ${ }^{66,67}$ Moreover, this real-time spectroscopy technique is expected to contribute to increased understanding of a wider class of phenomena in complex nonlinear systems. ${ }^{30}$

\section{Appendix A: Experimental Setup}

The schematic diagram of mode-locked laser is shown in Fig. 11. The laser system consists of a CNT-SA, a 3.5-m-long erbium-doped fiber (EDF) with $6 \mathrm{~dB} / \mathrm{m}$ absorption at $980 \mathrm{~nm}$, a polarization controller (PC), some single-mode fiber (SMF) pigtails, and a polarization-independent hybrid combiner of a wavelength division multiplexer, a tap coupler, and an isolator (WTI). The polarization-independent WTI is used to ensure the unidirectional operation, extract intracavity power with a ratio of $10 \%$, and input the pump power from the LD. An optical chopper is placed in the free-space section between LD and WTI to control the onset of solitons. CNT film acts as a mode locker to initiate the soliton operation. PC is utilized to optimize the mode-locking performance by adjusting cavity linear birefringence. The length of SMF is $\sim 4.3 \mathrm{~m}$. EDF and SMF have dispersion parameters of about 11.6 and $-22 \mathrm{ps}^{2} / \mathrm{km}$ at $1550 \mathrm{~nm}$, respectively. The total dispersion of laser cavity is about $-0.06 \mathrm{ps}^{2}$, and the total cavity length is $\sim 7.8 \mathrm{~m}$.

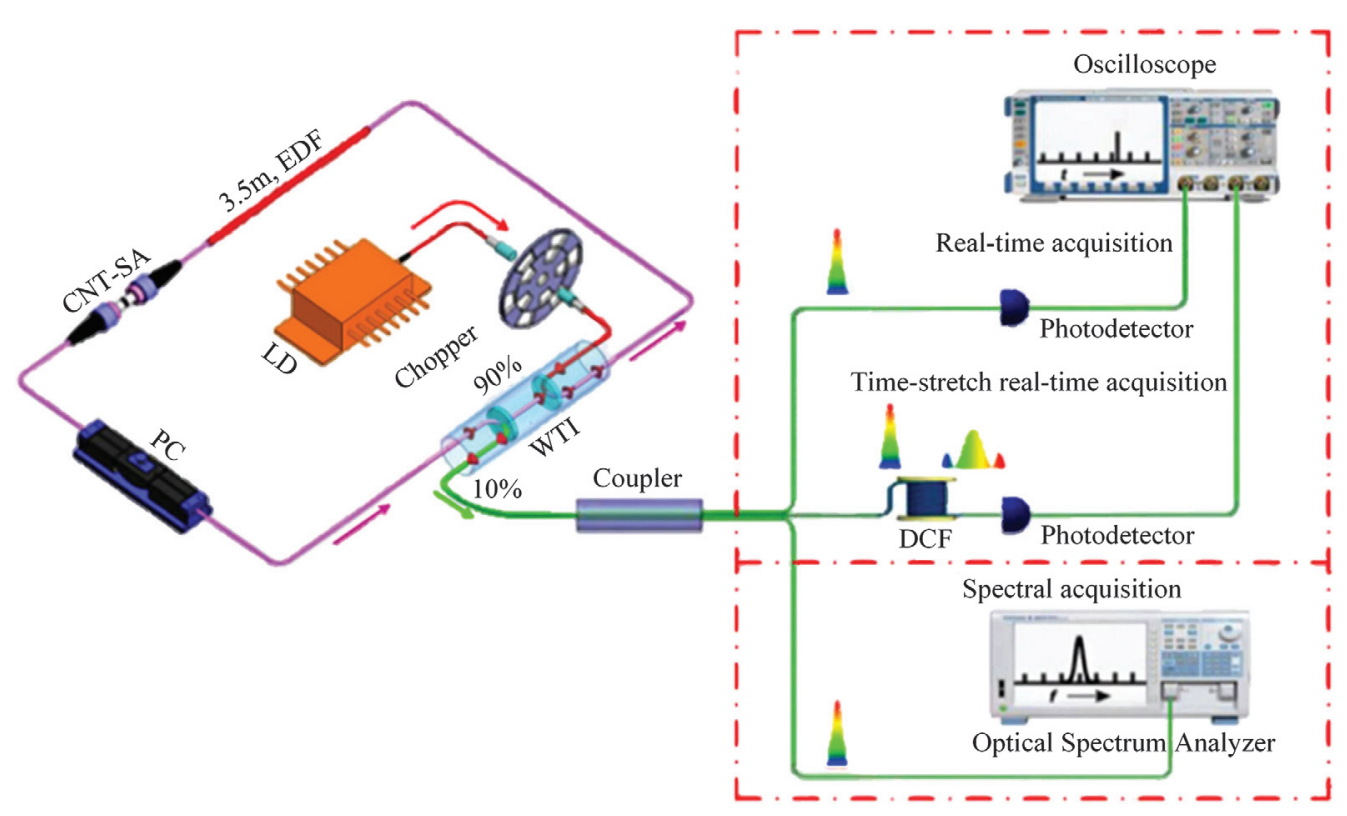

Fig. 11 Schematic diagram of the experimental setup for the mode-locked laser, generating the temporal solitons. The output can be characterized with real-time acquisition and time-averaged spectral acquisition (i.e., via optical spectrum analyzer). Real-time acquisition harnesses a highspeed real-time oscilloscope (20 GSa/s sampling rate) together with a high-speed photodetector. Timing data are achieved with direct detection, and real-time spectral data are obtained by dispersing the solitons in dispersion-compensating fiber (DCF) prior to detection. EDF, erbium-doped fiber; PC, polarization controller; LD, laser diode; CNT-SA, carbon nanotube saturable absorber; WTI, hybrid combiner of wavelength division multiplexer, tap coupler, and isolator. 
The real-time temporal and time-averaged spectral detections for solitons are recorded with a high-speed real-time oscilloscope and an OSA, respectively. The spectral information is mapped into the temporal waveform via DCF. The length of DCF is $\sim 5 \mathrm{~km}$ with the dispersion of about $-160 \mathrm{ps} /(\mathrm{nm} \cdot \mathrm{km})$.

\section{Appendix B: Relaxation Oscillation for Mode-Locked Laser}

The Kerr-lens lasers cannot usually self-start. ${ }^{1}$ In contrast, the laser here is mode-locked by the single-wall carbon nanotubes that serve as an excellent saturable absorber (i.e., mode locker) to self-start operation. The experimental setup is described above in detail. Some transient features at the nascent stage of this laser can be expressed by the rate equations, which provide a rather simple and intuitive picture of the lasing behavior. They can be written as ${ }^{55,65}$

$\frac{\mathrm{d} u}{\mathrm{~d} t}=R_{p}-u B_{s} q-\frac{u}{T_{u}}$,

$\frac{\mathrm{d} q}{\mathrm{~d} t}=u B_{s} q-\frac{q}{\xi}+\frac{u}{p_{m} T_{u}}$,

where $u$ and $q$ represent the population inversion and the photon number in the laser cavity, respectively. Here, $R_{p}$ is the pumping rate, $T_{u}$ is the lifetime of the upper-laser level, $\xi$ is the photon lifetime related to the cavity loss, $B_{s}$ is the Einstein coefficient given by $B_{s}=1 /\left(p_{m} T_{u}\right), p_{m}$ is the number of cavity modes coupled to the fluorescent line and is given by $p_{m}=8 \pi v^{2} \Delta \nu V / c^{3}$, where $v$ is the carrier frequency of photons, $\Delta \nu$ is the bandwidth of the laser medium, $c$ is the speed of light in vacuum, and $V$ is referred to as the mode volume within the laser cavity.

The total cavity loss, $\varsigma$, is time independent when the mode locker is absent in the laser cavity. Then, $\xi$ is a constant given by $\xi=L /(c \varsigma)$, where $L$ is the optical length of the laser resonator. Equations (5) and (6) can be used to describe the laser behaviors of both the $\mathrm{CW}$ and the transient operation, giving sufficiently accurate results for most practical purposes. On the contrary, $\varsigma$ is time-dependent when the mode locker (e.g., carbon nanotubes) is present in the laser cavity. The corresponding equation is expressed as $^{68}$

$\varsigma=\varsigma_{0}+\alpha_{0} /\left(1+I / I_{\text {sat }}\right)$,

where $\alpha_{0}, \varsigma_{0}$, and $I_{\text {sat }}$ are the linear limit of saturable absorption, nonsaturable loss, and saturation intensity, respectively. $I$ represents the input optical intensity, which is related to the photon number $q$ in the laser cavity. The relationship is given as $I=q \operatorname{ch} v / L$, where $h$ is the Planck's constant. ${ }^{55}$

For deriving Eqs. (5) and (6), we assume that the laser is oscillating on only one cavity mode. ${ }^{55}$ In the mode-locking regime, actually, the laser with the cavity length of $\sim 7.8 \mathrm{~m}$ generates pulses with the spectral bandwidth of $\sim 8.2 \mathrm{~nm}$, corresponding to about 40,000 locked modes. The stable mode-locking stage occurs when phases of various longitudinal modes are synchronized such that the phase difference between any two neighboring modes is locked to a constant value. ${ }^{64}$ Usually, pulses during the buildup process would not satisfy the ideal condition. ${ }^{69,70}$ Therefore, the deviations of the phase from the ideal values had been proposed by introducing a fluctuating background. ${ }^{71}$ During the self-starting process of the pulse laser, the mode locker can gradually drive thousands of modes from absolutely random state to the stable mode-locking state so that these cavity modes have regular phase relationships. The total optical field in the laser cavity can be expressed as ${ }^{72}$

$A(t)=\sum_{m=-M}^{M} A_{m} \exp \left(i \theta_{m}-i \rho_{m} t\right)$

where $A_{m}, \theta_{m}$, and $\rho_{m}$ are the amplitude, phase, and frequency of a specific mode among $2 M+1$ modes, respectively. The phase difference between any two neighboring modes is defined as $\theta=\theta_{m}-\theta_{m-1}$.

When the mode locker is present in the laser cavity, the laser parameters are employed in our simulations, including $R_{p}=3.75 \times 10^{16} \mathrm{~s}^{-1}$ (corresponding to the pump power of $\sim 16 \mathrm{~mW}), \quad T_{u}=10 \times 10^{-3} \mathrm{~s}, \quad \Delta \nu=4.68 \times 10^{12} \mathrm{~s}^{-1}, \quad V=$ $9 \times 10^{-10} \mathrm{~m}^{3}, \alpha_{0}=4.302 \%$, and $I_{\text {sat }}=9.67 \mathrm{MW} / \mathrm{cm}^{2}$. Here, $\theta$ is uniformly distributed on the interval from $-\theta_{0}$ to $\theta_{0}$, and $\theta_{0}$ is assumed as $4.8,4.2,3.65$, and 2.86 for the second to fifth laser spikes, as shown in Fig. 9(c), respectively. The phase differences of modes for the first laser spike distribute absolutely randomly. The simulation results are shown in Fig. 9(b), where Fig. 9(c) is the enlargement of all laser spikes. Each spike contains $\sim 300$ cavity roundtrips. Two insets in Fig. 9(c) are the local enlargements of the fourth and fifth laser spikes.

\section{Appendix C: Roundtrip Circulating-Pulse Method for Mode-Locked Laser}

Although Eqs. (5)-(8) can successfully predict the raised relaxation oscillation, they cannot describe the evolution and interaction of solitons in the stable mode-locking stage. We use a roundtrip circulating-pulse method to simulate the behaviors of beating and stable mode-locking stages. The numerical model includes the Kerr effect, the group velocity dispersion of fiber, the saturable absorption of mode locker, and the saturated gain with a finite bandwidth. ${ }^{62}$ When the pulses encounter cavity components, we take into account their effects by multiplying the optical field by the transfer matrix of a particular component. The simulation for the roundtrip circulating-pulse method starts with an arbitrary light field with the noise background [e.g., Fig. $10(\mathrm{~g})$ ]. After one roundtrip circulation in the laser cavity, the obtained results are used as the input of the next round of calculation until the light field becomes self-consistent. The simulation will approach to a stationary solution, which corresponds to a stable soliton solution of mode-locked laser under certain operation condition. When the optical pulses propagate through the fiber, the extended NSE is used to simulate the dynamics and evolution of the pulses, i.e., ${ }^{62}$

$\frac{\partial A}{\partial z}+i \frac{\beta_{2}}{2} \frac{\partial^{2} A}{\partial t^{2}}=\frac{g}{2} A+i \gamma|A|^{2} A+\frac{g}{2 \Omega_{g}^{2}} \frac{\partial^{2} A}{\partial t^{2}}$.

Here, $A, \beta_{2}$, and $\gamma$ represent the electric field envelop of the pulse, the fiber dispersion, and the cubic refractive nonlinearity of the fiber, respectively. The variables $t$ and $z$ are the time and the propagation distance, respectively. When the pulses propagate along the SMF, the first and last terms on the right-hand side of Eq. (9) are ignored. Here, $\Omega_{g}$ denotes the bandwidth 
of the gain spectrum and $g$ describes the gain function for the EDF and is given as ${ }^{62,73}$

$g=g_{0} \cdot \exp \left(-E_{p} / E_{s}\right)$

where $g_{0}, E_{p}$, and $E_{s}$ are the small-signal gain coefficient related to the doping concentration, the pulse energy, and the gain saturation energy that relies on pump power, respectively. To match the experimental conditions, we use the following parameters: $g_{0}=6 \mathrm{~dB} / \mathrm{m}, \quad \Omega_{g}=25 \mathrm{~nm}, \quad E_{s}=83 \mathrm{pJ}, \quad \gamma=$ $1.8 \mathrm{~W}^{-1} \mathrm{~km}^{-1}$ for EDF, and $\gamma=1 \mathrm{~W}^{-1} \mathrm{~km}^{-1}$ for SMF. Equation (9) is solved with a predictor-corrector split-step Fourier method. ${ }^{74}$

\section{Appendix D: Beginning Stage of the Birth of Solitons}

By using the extended NSE and roundtrip circulating-pulse method (see Sec. 8), we can simulate the evolution and interaction of solitons in the stable mode-locking stage and reveal the two different evolution processes of soliton buildup, as shown in Fig. 10. Some phenomena such as the Kelly sidebands [see Fig. 10(h)], transient bound state [see Fig. 10(f)], and beating pattern [see Fig. 10(b)] are achieved in the numerical calculations. Based on this method, however, an initial signal with the noise background has to be assumed in advance [see the red and black curves in Fig. 10(g)]. Figures 12 and 13 show the numerical results at the beginning stage of the birth of solitons, corresponding to the roundtrips from 1 to 62 . Figures 12(a) and 12(b) exhibit the beginning stage from 1 to 62 roundtrips for Figs. 10(a) and 10(c), respectively. Figures 13(a) and 13(b) demonstrate the beginning stage from 1 to 62 roundtrips for Figs. 10(d) and 10(f), respectively. By comparing Fig. 12 to Fig. 13, we can find that their beginning evolutions have slight differences due to the distinct initial signals. Obviously, the numerical results (see Figs. 12 and 13) are quite different from the experimental observations (see

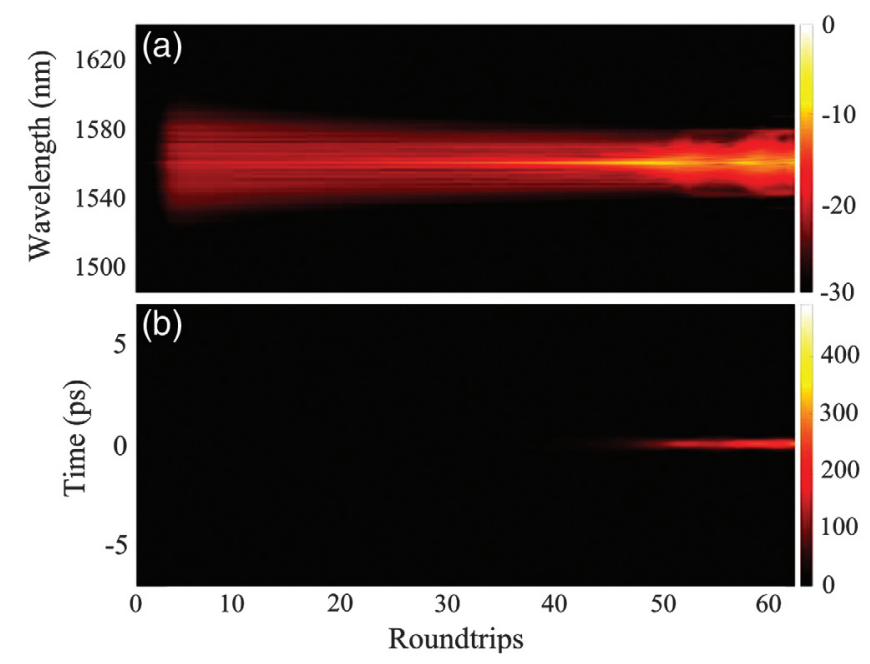

Fig. 12 Numerical results at the beginning stage (from 1 to 62 roundtrips) for the soliton buildup process with beating dynamics. (a) Spectral and (b) temporal evolutions of solitons along with roundtrips.

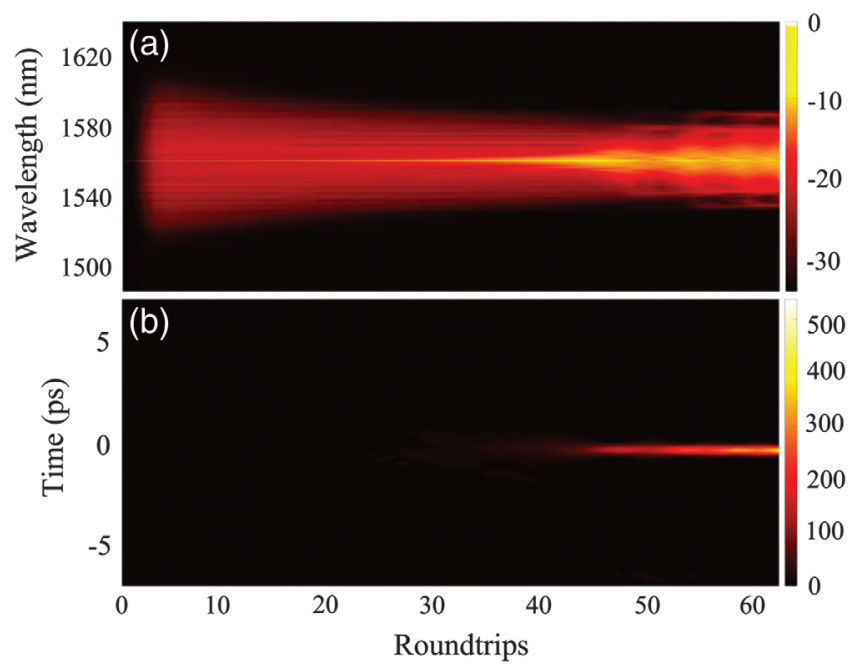

Fig. 13 Numerical results at the beginning stage (from 1 to 62 roundtrips) for the soliton buildup process with transient bound state. (a) Spectral and (b) temporal evolutions of solitons along with roundtrips.

Figs. 4 and 6). Therefore, the roundtrip circulating-pulse method together with extended NSE fail to achieve the raised relaxation oscillation at the beginning of the birth of solitons, although they can successfully describe two buildup pathways of solitons.

\section{Acknowledgments}

We thank X. Yao, X. Han, G. Chen, W. Li, G. Wang, and Y. Zhang for fruitful discussions. The work was supported by the National Natural Science Foundation of China under Grant Nos. 61525505, 11774310, and 61705193, by the Key Scientific and Technological Innovation Team Project in Shaanxi Province (2015KCT-06), and by China Postdoctoral Science Foundation (2017M610367).

\section{References}

1. G. Herink et al., "Resolving the build-up of femtosecond modelocking with single-shot spectroscopy at $90 \mathrm{MHz}$ frame rate," Nat. Photonics 10, 321-326 (2016).

2. M. Anderson et al., "Coexistence of multiple nonlinear states in a tristable passive Kerr resonator," Phys. Rev. X 7(3), 031031 (2017).

3. K. Goda, K. K. Tsia, and B. Jalali, "Serial time-encoded amplified imaging for real-time observation of fast dynamic phenomena," Nature 458, 1145-1149 (2009).

4. B. Li et al., "Panoramic-reconstruction temporal imaging for seamless measurements of slowly-evolved femtosecond pulse dynamics," Nat. Commun. 8, 61 (2017).

5. M. Pang et al., "All-optical bit storage in a fibre laser by optomechanically bound states of solitons," Nat. Photonics 10, 454-458 (2016).

6. H. Guo et al., "Universal dynamics and deterministic switching of dissipative Kerr solitons in optical microresonators," Nat. Phys. 13, 94-102 (2017).

7. M. Chernysheva et al., "Carbon nanotubes for ultrafast fibre lasers," Nanophotonics 6(1), 1-30 (2017).

8. P. J. Ackerman and I. I. Smalyukh, "Diversity of knot solitons in liquid crystals manifested by linking of preimages in Torons and Hopfions," Phys. Rev. X 7(1), 011006 (2017). 
9. J. M. Dudley et al., "Instabilities, breathers and rogue waves in optics," Nat. Photonics 8, 755-764 (2014).

10. E. Obrzud, S. Lecomte, and T. Herr, "Temporal solitons in microresonators driven by optical pulses," Nat. Photonics 11, 600-607 (2017).

11. P. Grelu and N. Akhmediev, "Dissipative solitons for mode-locked lasers," Nat. Photonics 6, 84-92 (2012).

12. Y. Wang et al., "Universal mechanism for the binding of temporal cavity solitons," Optica 4(8), 855-863 (2017).

13. M. Yu et al., "Breather soliton dynamics in microresonators," Nat. Commun. 8, 14569 (2017).

14. Q. Yang et al., "Counter-propagating solitons in microresonators," Nat. Photonics 11, 560-564 (2017).

15. X. Yi et al., "Single-mode dispersive waves and soliton microcomb dynamics," Nat. Commun. 8, 14869 (2017).

16. T. Dauxois and M. Peyrard, Physics of Solitons, Cambridge University Press, Cambridge (2015).

17. K. Krupa et al., "Real-time observation of internal motion within ultrafast dissipative optical soliton molecules," Phys. Rev. Lett. 118(24), 243901 (2017).

18. P. Marinpalomo et al., "Microresonator-based solitons for massively parallel coherent optical communications," Nature 546, 274-279 (2017).

19. M. G. Suh et al., "Microresonator soliton dual-comb spectroscopy," Science 354(6312), 600-603 (2016).

20. L. G. Wright, D. N. Christodoulides, and F. W. Wise, "Spatiotemporal mode-locking in multimode fiber lasers," Science 358(6359), 94-97 (2017).

21. J. Javaloyes, M. Marconi, and M. Giudici, "Nonlocality induces chains of nested dissipative solitons," Phys. Rev. Lett. 119(3), 033904 (2017).

22. M. Stratmann, T. Pagel, and F. Mitschke, "Experimental observation of temporal soliton molecules," Phys. Rev. Lett. 95(14), 143902 (2005).

23. F. Gustave et al., "Observation of mode-locked spatial laser solitons," Phys. Rev. Lett. 118(4), 044102 (2017).

24. D. Abraham et al., "Transient dynamics in a self-starting passively mode-locked fiber-based soliton laser," Appl. Phys. Lett. 63(21), 2857-2859 (1993)

25. C. Hönninger et al., " $Q$-switching stability limits of continuous wave passive mode locking," J. Opt. Soc. Am. B 16(1), 46-56 (1999).

26. L. Zinkiewicz, F. Ozimek, and P. Wasylczyk, "Witnessing the pulse birth-transient dynamics in a passively mode-locked femtosecond laser," Laser Phys. Lett. 10(12), 125003 (2013).

27. U. Keller, "Recent developments in compact ultrafast lasers," Nature 424, 831-838 (2003).

28. D. R. Solli et al., "Fluctuations and correlations in modulation instability," Nat. Photonics 6, 463-468 (2012).

29. D. R. Solli, J. Chou, and B. Jalali, "Amplified wavelength time transformation for real-time spectroscopy," Nat. Photonics 2, 48-51 (2008).

30. G. Herink et al., "Real-time spectral interferometry probes the internal dynamics of femtosecond soliton molecules," Science 356(6333), 50-54 (2017).

31. Y. Xu et al., "Ultrafast measurements of optical spectral coherence by single-shot time-stretch interferometry," Sci. Rep. 6, 27937 (2016).

32. D. R. Solli et al., "Optical rogue waves," Nature 450, 1054-1057 (2007).

33. T. Godin et al., "Real time noise and wavelength correlations in octave-spanning super continuum generation," Opt. Express 21(15), 18452-18460 (2013).

34. X. Wei et al., "Unveiling multi-scale laser dynamics through time-stretch and time-lens spectroscopies," Opt. Express 25(23), 29098-29120 (2017).

35. P. Ryczkowski et al., "Real-time full-field characterization of transient dissipative soliton dynamics in a mode-locked laser," Nat. Photonics 12, 221-227 (2018).
36. A. F. J. Runge, N. G. R. Broderick, and M. Erkintalo, "Dynamics of soliton explosions in passively mode-locked fiber lasers," J. Opt. Soc. Am. B 33(1), 46-53 (2016).

37. A. F. J. Runge, N. G. R. Broderick, and M. Erkintalo, "Observation of soliton explosions in a passively mode-locked fiber laser," Optica 2(1), 36-39 (2015).

38. M. Liu et al., "Successive soliton explosions in an ultrafast fiber laser," Opt. Lett. 41(6), 1181-1184 (2016).

39. A. Chong, L. Wright, and F. Wise, "Ultrafast fiber lasers based on self-similar pulse evolution: a review of current progress," Rep. Prog. Phys. 78(11), 113901 (2015).

40. E. Kelleher and J. Travers, "Chirped pulse formation dynamics in ultra-long mode-locked fiber lasers," Opt. Lett. 39(6), 1398-1401 (2014).

41. F. Krausz, T. Brabec, and C. Spielmann, "Self-starting passive mode locking," Opt. Lett. 16(4), 235-237 (1991).

42. E. P. Ippen, "Principles of passive mode locking," Appl. Phys. B 58(3), 159-170 (1994).

43. J. Herrmann, "Starting dynamic, self-starting condition and modelocking threshold in passive, coupled-cavity or Kerr-lens modelocked solid-state lasers," Opt. Commun. 98(1-3), 111-116 (1993).

44. J. M. Shieh et al., "Completely self-starting picosecond and femtosecond Kerr-lens mode-locked Ti:sapphire laser," J. Opt. Soc. Am. B 12(5), 945-949 (1995).

45. J. Solis et al., "Experimental study of a self-starting Kerr-lens mode-locked titanium-doped sapphire laser," Opt. Commun. 123(4-6), 547-552 (1996).

46. J. C. Kuo et al., "Pulse-forming dynamics of a cw passively modelocked Ti:sapphire/DDI laser," Opt. Lett. 17(15), 334-336 (1992).

47. N. W. Pu et al., "Starting dynamics of a cw passively mode-locked picosecond Ti:sapphire/DDI laser," Opt. Lett. 20(2), 163-165 (1995).

48. H. Li, D. G. Ouzounov, and F. W. Wise, "Starting dynamics of dissipative-soliton fiber laser," Opt. Lett. 35(14), 2403-2405 (2010).

49. Y. Yu et al., "Spectral-temporal dynamics of multipulse modelocking," Appl. Phys. Lett. 110(20), 201107 (2017).

50. J. Peng et al., "Real-time observation of dissipative soliton formation in nonlinear polarization rotation mode-locked fibre lasers," Commun. Phys. 1, 20 (2018).

51. J. Peng and H. Zeng, "Build-up of dissipative optical soliton molecules via diverse soliton interactions," Laser Photonics Rev. 12(8), 1800009 (2018).

52. X. Liu, X. Yao, and Y. Cui, "Real-time observation of the buildup of soliton molecules," Phys. Rev. Lett. 121(2), 023905 (2018).

53. Y. C. Tong, L. Y. Chan, and H. K. Tsang, "Fibre dispersion or pulse spectrum measurement using a sampling oscilloscope," Electron. Lett. 33(11), 983-985 (1997).

54. A. Mahjoubfar et al., "Time stretch and its applications," Nat. Photonics 11, 341-351 (2017).

55. O. Svelto, Principles of Lasers, Springer, New York (2010).

56. L. Larger, B. Penkovsky, and Y. Maistrenko, "Virtual chimera states for delayed-feedback systems," Phys. Rev. Lett. 111(5), 054103 (2013)

57. D. V. Churkin et al., "Stochasticity, periodicity and localized light structures in partially mode-locked fibre lasers," Nat. Commun. 6, 7004 (2015)

58. J. K. Jang et al., "Ultraweak long-range interactions of solitons observed over astronomical distances," Nat. Photonics 7, 657-663 (2013).

59. B. Garbin et al., "Topological solitons as addressable phase bits in a driven laser," Nat. Commun. 6, 5915 (2015).

60. A. Bednyakova and S. K. Turitsyn, "Adiabatic soliton laser," Phys. Rev. Lett. 114(11), 113901 (2015).

61. S. M. Kelly, "Characteristic sideband instability of periodically amplified average soliton," Electron. Lett. 28(8), 806 (1992).

62. X. Liu et al., "Distributed ultrafast fibre laser," Sci. Rep. 5, 9101 (2015). 
63. H. A. Haus and W. S. Wong, "Solitons in optical communications," Rev. Mod. Phys. 68(2), 423-444 (1996).

64. G. P. Agrawal, Nonlinear Fiber Optics, Academic Press, New York (2007).

65. R. Dunsmuir, "Theory of relaxation oscillations in optical masers," Int. J. Electron. 10(6), 453-458 (1961).

66. M. Leonetti, C. Conti, and C. Lopez, "The mode-locking transition of random lasers," Nat. Photonics 5, 615-617 (2011).

67. T. Herr et al., "Temporal solitons in optical microresonators," Nat. Photonics 8, 145-152 (2014).

68. X. Liu et al., "Versatile multi-wavelength ultrafast fiber laser mode-locked by carbon nanotubes," Sci. Rep. 3, 2718 (2013).

69. A. A. Grutter, H. P. Weber, and R. Dandliker, "Imperfectly modelocked laser emission and its effects on nonlinear optics," Phys. Rev. 185(2), 629-643 (1969).

70. R. H. Picard and P. Schweitzer, "Theory of intensity correlation measurements of imperfectly mode-locked lasers," Phys. Rev. A 1(6), 1803-1818 (1970).

71. R. Dandliker, H. P. Weber, and A. A. Grutter, "Influence of systematic phase deviations on the output of mode-locked lasers," Z. Angew. Math. Phys. 20(4), 572-574 (1969).

72. G. P. Agrawal, Applications of Nonlinear Fiber Optics, Academic Press, New York (2008).
73. G. P. Agrawal, "Amplification of ultrashort solitons in erbiumdoped fiber amplifiers," IEEE Photonics Technol. Lett. 2(12), 875-877 (1990).

74. X. Liu and B. Lee, "A fast method for nonlinear Schrodinger equation," IEEE Photonics Technol. Lett. 15(11), 1549-1551 (2003).

Xueming Liu is a professor at the College of Optical Science and Engineering, Zhejiang University. He received his $\mathrm{PhD}$ from the Department of Electronic Engineering, Southeast University, Nanjing, China, in 2000. His current research interests include nonlinear optics, fiber lasers, and ultrafast optics.

Yudong Cui is an assistant research fellow at the College of Optical Science and Engineering, Zhejiang University. He received his PhD in optical engineering from the University of Chinese Academy of Sciences. From 2016 to 2018 , he was engaged in postdoctoral research at Zhejiang University, and then joined the College of Optical Science and Engineering at Zhejiang University. His current research interests include nonlinear optics of nanomaterials, high-energy ultrafast fiber lasers, and related applications. 